\title{
Correction to: A compressive mathematical model including an eclipse stage of infected cells to study the impact of stem cells transplantation on one HIV1 patient
}

\author{
Saoussan Kallel-Jallouli ${ }^{1}$. S Sundus Naji Alaziz $^{2}$ (D)
}

Published online: 21 February 2022

(c) Springer-Verlag GmbH Austria, part of Springer Nature 2022

\section{Correction to: \\ Network Modeling Analysis in Health Informatics and Bioinformatics \\ https://doi.org/10.1007/s13721-020-00282-3}

Unfortunately, the corresponding author's affiliation was published incorrectly in the online published article. The correct affiliation is given below.

Faculty Mathematical Sciences, College of Science, Princess Nourah bint Abdulrahman University, Riyadh, Saudi Arabia.
Publisher's Note Springer Nature remains neutral with regard to jurisdictional claims in published maps and institutional affiliations.

The original article can be found online at https://doi.org/10.1007/ s13721-020-00282-3.

Sundus Naji Alaziz

snalaziz@pnu.edu.sa

1 LR03ES04, Faculté des Sciences de Tunis, Université de Tunis el Manar, 2092 Tunis, Tunisia

2 Faculty Mathematical Sciences, College of Science, Princess Nourah bint Abdulrahman University, Riyadh, Saudi Arabia 